\section{Erythropoese, ineffektive}

H. Baum

Institut für Laboratoriumsmedizin, Mikrobiologie und Blutdepot, Regionale Kliniken Holding RKH GmbH, Ludwigsburg, Deutschland

Englischer Begriff ineffective eythropoiesis

Definition Verminderung der effektiv produzierten Erythrozyten bei ausreichender Anzahl erythrozytärer Vorstufen im Knochenmark.

Beschreibung Die ineffektive Erythropoese beschreibt Zustände, bei denen die Zellreifung im Proliferations- und
Reifungskompartiment der Erythropoese gestört ist. Während der Proliferations- und Reifungsphase geht ein Großteil der - Erythroblasten wieder zugrunde mit dem Ergebnis einer ungenügenden Anzahl an reifen Erythrozyten. Bei intaktem Regulationskompartiment kommt es intramedullär zu einer regulativen Erhöhung der Anzahl unreifer Erythroblasten mit erhöhter Markzelldichte.

\section{Literatur}

Heimpel H, Prümmer O (1998) Bedeutung und Effizienz der Blutzelldiagnostik. In: Boll I, Heller S (Hrsg) Praktische Blutzelldiagnostik. Springer, Berlin/Heidelberg/New York, S 6-35 